\title{
¿Geografía o Ciencias Sociales como asignatura en la enseñanza secundaria?
}

\author{
antonio Zarate Martín
}

La posible pérdida de personalidad de la geografía, integrándose dentro de una asignatura de ciencias sociales, es un tema de actualidad en nuestro sistema educativo y de hondas repercusiones en la formación de los futuros ciudadanos. Es una cuestión de debate que se plantea en el contexto de la reforma de la enseñanza no universitaria en España y dentro del avance o retroceso de la geografía con relación a las ciencias sociales, consideradas como una asignatura en los currículos escolares de algunos países. ¿La geografía es una disciplina singular, con carga de contenidos propios y utilidad social para mantener el papel tradicional de asignatura autónoma y troncal, o debe ser absorbida en el marco amplio de las ciencias sociales como materia académica globalizadora? Es manifiesto que ambas posturas cuentan con defensores. Nosotros nos apresuramos a señalar que nos incluimos entre los partidarios de mantener su independencia, al menos en la enseñanza secundaria, por las razones que se irán señalando.

\section{AUGE DE LAS CIENCIAS SOCIALES Y REPLIEGUE DE LA GEOGRAFIA}

Para una aproximación a esta problemática hay que destacar el desarrollo creciente de las ciencias sociales desde los años siguientes a la 
Segunda Guerra Mundial a nuestros dias, su incorporación a los currículos escolares y el retroceso de la geografía como asignatura autónoma en muchos países durante ese mismo período de tiempo. Las razones de estos hechos se hallan en:

\section{Prestigio reciente de la economía y sociología}

Estas ciencias sociales se desarrollan a partir de los años 30 y 40 , impulsadas por el positivismo lógico, y se revalorizan tras la Segunda Guerra Mundial, cuando aparecen como ciencias útiles para las necesarias tareas de división regional, planificación del territorio, reconstrucción de ciudades y áreas devastadas, y para atender a los problemas de subdesarrollo. Economistas y sociólogos se mostraron más capacitados y mejor preparados que los geógrafos para estas labores. Paradójicamente, figuras como Von Thünen, Weber o Losch supieron recoger y aplicar desde sus disciplinas, antes que los geógrafos, las teorias sobre regionalización y organización del espacio de Christaller.

Los economistas y sociólogos incorporaron técnicas de análisis y conceptos de espacio económico homogéneo, espacio polarizado y región-plan que más tarde utilizaron los geógrafos.

Todo ello fue facilitado por la revolución tecnológica que posibilitó el tratamiento de una información progresivamente más abundante, y por el neopositivismo, en el que se inscriben la teoría general de los sistemas, teorías de la información y de la comunicación, teorías de la decisión y de los juegos, y la construcción de modelos. La consecuencia fue un enorme prestigio y reconocimiento social hacia estas ciencias.

\section{Deseo de ajustar el sistema educativo a nuevas necesidades de la sociedad}

Después de la década de los cincuenta se manifiesta en todas partes una inadecuación entre el sistema educativo tradicional y las exigencias de la estructura productiva, las necesidades de escolarizar a una gran cantidad de niños, generada por el "baby boom" de posguerra, el deseo de proporcionar a un mayor número de personas el acceso a las enseñanzas secundaria y universitaria, y el afán de facilitar su incorporación al mundo laboral. Se impone así una renovación de los currículos y de los métodos de aprendizaje. La finalidad de la educación deja de 
ser la de transmitir básicamente conocimientos para preocuparse también por objetivos de comportamiento, y en cuanto a los contenidos, se priman los de carácter científico y utilitarios sobre las humanidades. Se sustituye la memorización y la acumulación de información por el desarrollo de técnicas y habilidades según el principio de aprender investigando. Se pretende formar ciudadanos que sepan desenvolverse por si mismos, que puedan hacer frente a las situaciones concretas y problemas de la vida, y que sean capaces de integrarse en el nuevo mercado de trabajo. En esta coyuntura, muchas de las disciplinas existentes resultan obsoletas, entre ellas la geografía; mientras, las ciencias sociales se muestran más acordes con las exigencias de los nuevos tiempos. Se presentan como medio de conocimientos de la realidad social a partir de la cual se puede ejercer una actitud crítica respecto a ella ${ }^{1}$.

Por otra parte, la crisis económica que se abre en los años setenta, con los problemas de paro, desempleo y reconversión productiva, también repercute en el sistema educativo. La sociedad reclama más una formación profesional que educativa. Se prefieren materias como matemáticas, ciencias físicas, economía o lenguas vivas, susceptibles de aplicarse a la vida laboral, a asignaturas tradicionales como la geografía o la historia. Estas últimas se ven forzadas a ceder parte del tiempo del que disponían antes en la enseñanza.

\section{Problemas de la propia geografía}

El agotamiento del paradigma vidaliano y con él de la región geográfica, de carácter holístico, que proporcionaba un objeto exclusivo de análisis de la geografía, produce desorientación, justo en la época en que sociología y economía se afirman como ciencias útiles. Simultáneamente se abre una etapa de crispación en la que nuevas corrientes de geografía, relacionadas con el pensamiento científico del momento, intentan recuperar para nuestra disciplina el valor que tuvo en el pasado.

La geografía cuantitativa, al socaire del neopositivismo, cuyo origen se encuentra en el círculo de Viena, sobre todo en el discurso filosófico de Neurath ${ }^{2}$, y se desarrolla en los países anglosajones durante las dé-

\footnotetext{
' V. Garcia Hoz, Elementos para un programa de Enseñanzas Sociales. Biblioteca de Educación Personalizada, Valladolid. Ed. Miñón, 1971.

${ }^{2}$ H. CAPEL, Filosofía y Ciencia en la Geografía Contemporánea. Barcelona. Ed. Barcanova, 1981 , p. 367-370.
} 
cadas de los años cuarenta y cincuenta, se presenta como ciencia nomotética que busca establecer leyes, descubrir regularidades y principios generales que rigen un orden espacial ahistórico, dentro de una concepción monista de la realidad y de un reduccionismo fisicalista.

La quiebra del neopositivismo, los movimientos críticos y radicales de finales de los sesenta, y corrientes filosóficas como la fenomenología y el existencialismo conducen a la geografia a la recuperación de la herencia histórica y la comprensión frente a la explicación, el descubrimiento de la dimensión subjetiva, la valoración de la experiencia personal y del espacio como producto social. Dentro de estas corrientes se hallan la geografía de la percepción y del comportamiento, la geografía humanística y la geografia radical.

Todas estas tendencias proporcionan nuevos enfoques, planteamientos y técnicas. Son las bases sobre las que se ha operado en muchos países la recuperación de la geografía, con un reflejo no exento de importancia en los currículos escolares.

\section{RENOVARSE O MORIR}

Hoy, en casi todos los países la geografía se halla integrada en áreas de conocimiento multidisciplinar entre los 6 y 9 años, bien medioambientales 0 de carácter científico social, incluso en algunos paises esta integración se extiende a lo largo de toda la enseñanza secundaria. Sin embargo, la tradición de la geografía en los currículos, el esfuerzo de renovación y adaptación a la nueva coyuntura histórica y a las exigencias educativas de la sociedad, y su vinculación con la enseñanza superior, han permitido que la geografía permanezca en la mayoría de los países como materia independiente, obligatoria u optativa en casi todos los cursos de la enseñanza secundaria ${ }^{3}$.

Hasta mediados de los años sesenta, los objetivos y contenidos de la geografía en secundaria eran bastante tradicionales. La geografía era una descripción explicativa de los paisajes mediante el método inductivo. Se partía de la realidad, de fotografías, mapas a gran escala y descrip-

${ }^{3}$ V. CAPEL, A. LuIs y L. URTEAGA, “La Geografía ante la Reforma Educativa". Geocritica, $\mathrm{n}^{\circ}$ 53, Barcelona 1984. 
ciones estadisticas. En gran manera eran resultado del modelo vidaliano y de una herencia trasnochada del siglo XIX, cuando la geografía gozó de mayor popularidad. Se estimulaba el conocimiento del propio país en un discurso político ideológico de raíces nacionalistas, se fomentaba el respeto a la naturaleza y se procuraba el conocimiento de otros pueblos.

A partir de entonces se afirman objetivos comportamentales dirigidos al desarrollo ciudadano del individuo, a su emancipación, participación, fomento de su capacidad de análisis y crítica, y a su independencia en la toma de decisión. En cuanto a los contenidos, se tiende a disminuir la orientación regional y se insiste en aspectos sistemáticos y de reflexión espacial sobre los grandes problemas del mundo, sobre todo en los paises más innovadores. Se propone el estudio del medio a través de círculos concéntricos que van de la realidad local al planeta en su conjunto, y se recomienda el análisis de casos como resultado de la necesidad de reducir contenidos en los programas sin pérdida de profundidad en el tratamiento de los temas. Se destaca la dimensión espacial de los fenómenos geográficos y se introducen conceptos generales en relación con la teoría de los sistemas.

Se presta especial atención al proceso de aprendizaje dentro de un modelo educativo eficiencista que disminuye la información y potencia las actividades y el interés por la estructura de la ciencia. La renovación metodológica estimula la clase activa, las excursiones, el trabajo de campo, las pesquisas, las investigaciones, los juegos de simulación, las técnicas audiovisuales y el uso del ordenador.

Los currículos abiertos, flexibles y no normativos, que estuvieron de moda durante algunos años, han dejado paso en casi todas partes a programas elaborados, con instrucciones muy detalladas y de obligado cumplimiento. En este sentido ha habido una reacción análoga a la que se empieza a producir con relación a los excesos derivados del estudio del medio local, a partir del cual algunos pretenden mostrar toda la problemática geográfica del mundo actual.

\section{EN ESPAÑA, POCAS CIENCIAS SOCIALES Y ESCASA GEOGRAFÍA}

La Ley General de Educación de 1970 introdujo en España el término ciencias sociales como título de una asignatura y limitó la geografía en el Bachillerato a una mínima presencia. Esta Ley representaba en 
nuestro país la corriente reformista e innovadora dirigida a la adecuación del sistema educativo a las nuevas demandas sociales, científicas y pedagógicas, a la necesidad de conectar la enseñanza con el aparato productivo y a facilitar para ello el acceso de la población a la enseñanza media y superior. Dentro del contexto general de renovación del sistema educativo y siguiendo directrices de la UNESCO y de modelos anglosajones, planteaba la modernización científica de la educación. Se primaban materias de tipo instrumental y científico en perjuicio de las humanidades. Se introducía una asignatura con la denominación de ciencias sociales en el primer nivel de enseñanza, EGB, que se ampliaba a costa del Bachillerato y se hacía obligatorio hasta los 14 años. La geografía dejaba de existir como disciplina independiente en ese nivel integrándose dentro de las ciencias sociales. Sin embargo, en la práctica, a pesar del título de la asignatura y de la declaración de intenciones, las ciencias sociales no han pasado de ser una simple yuxtaposición de temas de antiguas asignaturas: geografia, historia y educación civica.

Por otro lado, la geografía se limitaba en el nuevo Bachillerato, BUP, a una asignatura de geografía humana y económica en el segundo curso y a una asignatura de tercero, compartida con historia de España y de los países hispánicos, y durante unos años con la enseñanza de la Constitución. En COU hubo una Geografía Económica que duró poco tiempo.

Por si fuera poco, los escasos contenidos de geografía de BUP son reiterativos de los de EGB, lo que favorece el aburrimiento y desinterés de los alumnos por nuestra materia.

La pérdida de entidad de la geografia en el sistema educativo español se explica por los factores generales señalados antes y por aspectos particulares como son:

- Pequeño interés de la Universidad por la enseñanza de la geografía en otros niveles a diferencia de lo que se puede observar en el resto de Europa. Es un hecho favorecido por un sistema educativo que establece una profunda separación entre la enseñanza universitaria y no universitaria. La desconexión repercute también en la falta de actualización de objetivos, contenidos y técnicas de trabajo en EGB y BUP.

Los profesores de Universidad se sienten más preocupados por abrir camino a la geografía en ordenación del territorio, donde arquitectos, economistas y sociólogos ocupan posiciones consolidadas profesional e institucionalmente. En cambio, no muestran interés por lo que ha constituido la salida profesional más inme- 
diata y que hoy empiezan a disputar demógrafos, ecólogos, sociólogos y economistas.

- Escasa formación geográfica de la mayor parte de los profesores que enseñan esta disciplina en los niveles no universitarios:

En BUP, predominan profesores de formación histórica y no son pocos los que pertenecen a asignaturas distintas pero son obligados a enseñar geografía para completar su horario. Es un problema que todavía puede agravarse más con el nuevo proyecto de reforma de la enseñanza, en el caso de que los profesores de la etapa obligatoria de secundaria dejen de ser especialistas de materias diferentes para convertirse en profesores de áreas de conocimiento.

En EGB, la enseñanza de la geografía fue asumida por profesores que, en el mejor de los casos, eran magníficos pedagogos, pero carecian de formación adecuada para una disciplina que antes era impartida por licenciados.

Como consecuencia de todo ello los alumnos no son motivados suficientemente, se aburren y rechazan la geografía como un conocimiento inútil y sin sentido.

- Reducida sensibilidad de las autoridades ministeriales hacia la geografía. Nuestra disciplina tiene menor presencia en los proyectos actuales de reforma de la enseñanza que en cualquiera de los otros países de nuestro entorno, como Francia, República Federal Alemana, Reino Unido o Portugal, a pesar de la conveniencia de aproximar nuestro sistema educativo a sus curriculos escolares para facilitar la integración que impondrá la aplicación del Acta Única de la Comunidad Económica Europea.

Como unas manifestaciones más de este desinterés por la geografía, el Ministerio de Educación y Ciencia ha propiciado este mismo año la desaparición de esta asignatura del Bachillerato Internacional, la ha suprimido de las pruebas de Selectividad para alumnos que cursan estudios en el extranjero y la ha reducido a una materia optativa en el curso de acceso a la Universidad para mayores de 25 años.

- Desfase entre las investigaciones científica y didáctica y la práctica de la enseñanza. Es consecuencia de la desconexión Universidad-Escuela, que impide la difusión de ideas nuevas, y de la ineficacia de los programas y sistemas ministeriales de actualización y formación del profesorado, entre ellos los CEPS. La mayo- 
ría carecen de propuestas globales de programación, se mueven en un horizonte teórico didáctico reducido y apenas pasan de vagas formulaciones de principios en favor de la enseñanza activa.

La actualización de los enseñantes se basa en el mero voluntarismo, en la improvisación de experiencias, en prácticas de aprendizaje no contrastadas, a diferencia de otros países, donde el perfeccionamiento del profesorado ocupa un lugar importante de la política presupuestaria y educativa, y se realiza de modo eficaz a través de los servicios de la inspección técnica y de cursos a tiempo completo o parcial durante uno o dos años en los centros universitarios.

\section{MÁS GEOGRAFÍA PARA UNA MEJOR COMPRENSIÓN DE UN MUNDO COMPLEJO}

Es en esta situación y de cara a las exigencias educativas de la sociedad, a la plena incorporación a la Comunidad Europea y a la reforma de los planes de estudio, cuando adquieren verdadero sentido las cuestiones en torno al papel que debe corresponder a la geografía en los nuevos currículos y la conveniencia de integrarse dentro del área de ciencias sociales o mantener su tradicional autonomía. El momento no puede ser más interesante, pues bien puede ser la ocasión para que la geografía actualice objetivos, contenidos, técnicas de aprendizaje, y recupere el valor troncal que tuvo en España desde la Ley Moyano (1857) a la aprobación de la Ley General de Educación ${ }^{4}$.

Si se trata de acercarnos a los planes de estudio del entorno europeo parece fuera de toda duda que la geografía debe contar con una mayor representación curricular en la enseñanza y que sería conveniente mantener su autonomía, al menos desde los 12 a los 18 años, como ocurre en otros países. Por otra parte, las razones para aconsejar su independencia respecto a su integración en el área de ciencias sociales son variadas:

1. La geografía incorpora aspectos espaciales que corresponden a las ciencias sociales y a las ciencias de la naturaleza. Es una

${ }^{4}$ A. LuIs, La Geografía del Bachillerato Español (1836-1870). Historia de una crisis. E. Universidad de Barcelona, 1986. 
disciplina puente entre unas y otras. De este modo, resulta más adecuada para comprender el mundo actual en su complejidad y analizar las interrelaciones de sus distintos elementos que las ciencias sociales. Lacoste ${ }^{5}$ dice "La geografia es una bisagra entre el conocimiento de los hechos físicos, es decir, la naturaleza, y el de los hechos humanos".

2. Las ciencias sociales no existen como asignatura con campo estructurado de conocimiento, ni de teoría científica ni de método de indagación. Cubren un amplio campo de materias para cuya variedad resulta difícil encontrar profesorado adecuado. Por el contrario, como señala Claval ${ }^{6}$, la geografía es una disciplina concreta, palpable y sensitiva, de valores casi materiales que puede ser enseñada desde el parvulario a la Universidad; lo que no sucede con el derecho, la sociología, la antropologia, la economía...

3. La justificación didáctica de las ciencias sociales se efectúa sobre la base de una pretendida interdisciplinariedad entre diferentes materias para hacer comprender al alumno una realidad que es compleja y que el alumno percibe como única en su mente, sobre todo en los primeros niveles. Sin embargo, los programas de sociales son una simple yuxtaposición de asignaturas distintas, mientras que la geografía es en si misma una ciencia o saber, en términos de Lacoste ${ }^{7}$, que tiene como objetivo estudiar el entrecruzamiento de diferentes disciplinas en el espacio. De este modo, se muestra como el saber más adecuado para captar la complejidad de la realidad, y en ello mismo radica parte de su utilidad social.

Para aumentar la presencia de la geografia en los planes de estudio y garantizar su independencia es preciso convencer a la opinión pública de su utilidad y capacidad para contribuir a una mejor formación de los ciudadanos y a una mayor comprensión de los problemas del planeta, a parte de hacer ver su eficacia en el desarrollo de las aptitudes men-

5 Y. LACOSte, "La Geografia", en F. Chatelet, Historia de la Filosofia. Ideas y Doctrinas. Espasa Calpe, vol. 4, 1976, p. 218-272.

'P. Claval y Y. Guermond, «Débat: L'enseigement de la Géographie». Espace Géographique, n. 1 , t. XV, 1986, p. 5-13.

7 Y. LACOSTE, La enseñanza de la Geografía. Universidad de Salamanca, Documentos Didácticos, 1986. 
tales ${ }^{8}$. Como indica Lacoste ${ }^{9}$, la geografía debe preparar para la acción y en este sentido hay que acabar con la actual dicotomía entre geografía en la escuela y en el mundo exterior. Graves ${ }^{10}$ dice: "la única diferencia que se ha de plantear entre los enseñantes de geografía es la que separa a los que piensan que la geografia sirve para abrir el espíritu del estudiante al mundo exterior, tal como es, y los que creen que la geografia debe ayudar a transformar el mundo exterior".

La geografía es una forma de razonamiento, un saber pensar el espacio que permite más eficacia en la actuación, de ahí la importancia que siempre la han concedido los militares y el valor que hoy la otorgan las multinacionales, como señala Lacoste ${ }^{11}$; unos y otras utilizan su estrategia para definir sus líneas de actuación y control político y económico del mundo. Referencias a la acción, al dominio militar, a las disputas de mercados entre multinacionales son aspectos del mundo actual que la geografía considera y que habría que incorporar a la escuela para que nuestra disciplina no sea exclusiva de unos pocos que la utilizan en beneficio de sus intereses.

Ahora bien, para conseguir estos objetivos y recuperar en la escuela el significado y utilidad que la geografía tiene en el mundo actual, es necesaria una enseñanza de calidad basada en:

- Calidad del profesor. La calidad de la enseñanza de la geografía depende más del nivel del enseñante que de las técnicas de aprendizaje. En este sentido es primordial realizar un esfuerzo para facilitar la tarea de los profesores deseosos de enseñar geografía moderna y para conseguir su actualización. La geografía debe ser enseñada por profesores que posean un verdadero sentido del espacio.

- Elaboración meditada de un nuevo currículo de la geografia en la enseñanza no universitaria que asuma corrientes, tendencias y técnicas actuales de la geografía en relación con los problemas de nuestro mundo. Hay que introducir los avances científicos y técnicos de los últimos años y es preciso evitar reiteración y yux-

" T. BENNETS, "The nature of geographical objectives", en R. L. WALFORD, News directions in Geography teaching. Longman, 1973.

"Y. LACOSTE, "Débat: L'enseignement de la Géographie", en P. Claval y Y. Guermond, Espace Géographique, n. ${ }^{\circ} 1$, t. XV, 1986, p. 5-13.

${ }^{10}$ N. Graves, “La Géographie dans l'enseignement sécondaire, l'experience anglaise». L'Espace Géographique, n. ${ }^{\circ}$, t. XV, 1986, p. 131-137.

1 Y. LACOSTE, La Geografia, un arma para la guerra. Barcelona. Ed. Anagrama, 1977. 
taposición de contenidos como ocurre entre los programas actuales de EGB y BUP. Convendría incorporar tensiones y elementos dramáticos que están presentes en los conjuntos espaciales y que constituyen también objeto de la geografía.

- Valoración de las posibilidades y ventajas formativas de las diferentes corrientes geográficas para integrarlas en propuestas didácticas adecuadas a los objetivos y necesidades de los alumnos, según su edad y desarrollo cognitivo. La enseñanza de la geografía ha de saber crear modos de pensar el espacio y ha de capacitar al alumno para que de alguna medida sea geógrafo por sí mismo.

\section{CONCLUSIÓN: GEOGRAFÍA COMO ASIGNATURA INDEPENDIENTE}

A la vista de todo lo argumentado podemos concluir recogiendo las palabras de Anuchin: «Es preciso contar con una ciencia que se preocupe del estudio de la utilización de la naturaleza y que conecte las ciencias naturales con el grupo de las ciencias sociales: jlo que necesitamos es una geografía sin adjetivos!» ${ }^{12}$, al menos para los niveles educativos situados por encima de los 11-12 años, teniendo en cuenta las teorías de Piaget sobre psicología evolutiva que fijan en esa edad el paso del pensamiento concreto al pensamiento formal ${ }^{13}$. De todas formas, es preciso señalar que la geografía encierra una alta carga de concreción, los mismos mapas son representaciones abstractas concretas, por lo que estimamos que en manera alguna debe quedar descalificada para ser impartida como asignatura independiente en las primera etapas de la educación; en realidad es lo que se está haciendo ahora mismo en los ciclos inicial y medio, aunque yuxtapuesta a temas de historia y educación civica.

Lo cierto es que la comprensión de la complejidad del mundo actual necesita un instrumento de análisis que proporciona mejor la geografia que una amalgama de ciencias humanas, en las que la preocupación por

12 V. A. ANuchin, "Teoría de la Geografía", en R. Chorley, Nuevas tendencias en Geografia. Madrid, IEAL, cap. III, p. 97, 1975.

13 J. PIAGET, Seis estudios de Psicología. Barcelona. Ed. Seix Barral, 1977. 
el espacio, las intersecciones y entrecruzamientos de los fenómenos estudiados por cada una de ellas se pierden. En definitiva, si se pretende la interdisciplanariedad, ninguna ciencia está mejor preparada para ello que la geografía. 